\title{
Essential Oils from the Malaysian Citrus (Rutaceae) Medicinal Plants
}

\author{
Siti Nur Atiqah Md Othman ${ }^{1}$, Muhammad Aizam Hassan ${ }^{1}$, Lutfun Nahar ${ }^{2}$, Norazah Basar ${ }^{1, *}$, \\ Shajarahtunnur Jamil ${ }^{1}$ and Satyajit D. Sarker ${ }^{2}$ \\ 1 Department of Chemistry, Faculty of Science, Universiti Teknologi Malaysia, 81310 Johor Bahru, Johor, \\ Malaysia; snamo66@yahoo.com (S.N.A.M.O.); mdzam91@gmail.com (M.A.H.); shaja@kimia.fs.utm.my (S.J.) \\ 2 Medicinal Chemistry and Natural Products Research Group, School of Pharmacy and Biomolecular Sciences, \\ Liverpool John Moores University, James Persons Building, Byrom Street, Liverpool L3 3AF, UK; \\ 1.nahar@ljmu.ac.uk (L.N.); s.sarker@ljmu.ac.uk (S.D.S.) \\ * Correspondence: norazah@kimia.fs.utm.my; Tel.: +607-5534345; Fax: +607-5566162
}

Academic Editor: Ronan Batista

Received: 18 January 2016; Accepted: 16 May 2016; Published: 3 June 2016

\begin{abstract}
This review article appraises the extraction methods, compositions, and bioactivities of the essential oils from the Citrus species (family: Rutaceae) endemic to Malaysia including C. aurantifolia, C. grandis, C. hystrix, and C. microcarpa. Generally, the fresh peels and leaves of the Citrus species were extracted using different methods such as steam and water distillation, Likens-Nikerson extraction, solvent extraction, and headspace solid-phase micro-extraction (HS-SPME). Most of the Citrus oils were found to be rich in monoterpene hydrocarbons with limonene (1) as the major component identified in the peels of C. aurantifolia (39.3\%), C. grandis ( $81.6 \%-96.9 \%)$, and C. microcarpa $(94.0 \%)$, while sabinene (19) was the major component in the peels of $C$. hystrix $(36.4 \%-48.5 \%)$. In addition, citronellal (20) (61.7\%-72.5\%), linalool (18) (56.5\%), and hedycaryol (23) (19.0\%) were identified as the major components in the oil of C. hystrix leaves, C. grandis blossom and C. microcarpa leaves, respectively. The $C$. hystrix essential oil has been experimentally shown to have antimicrobial and antifeedant activities, while no bioactivity study has been reported on the essential oils of other Malaysian Citrus species.
\end{abstract}

Keywords: Citrus; Rutaceae; essential oils; extraction; composition; bioactivities

\section{Introduction}

The Citrus species of the family Rutaceae provide several popular edible fruits in the world. The species are widely distributed in the Indo-Malaysia region, South-East Asia, India and China, but cultivated worldwide [1]. The fruits and the leaves of the Citrus species contain a variety of essential oils with various distinct flavors, and biologically-active compounds, which are important to human nutrition and diet, which include vitamin C, folic acid, potassium, flavonoids, coumarins, pectin, and dietary fibers. In Malaysia, the oils from the fruits and the leaves are commercially used as flavors and fragrances, as well as in cooking, perfumery and medical treatments, especially in aromatherapy [2]. Recent studies on the Malaysian Citrus plants have reported the identification and composition of essential oils of several Citrus species including C. aurantifolia, C. grandis, C. hystrix, and C. microcarpa. A monoterpene hydrocarbon, limonene (1), is the major component in the essential oils from the peels of these Malaysian Citrus species [3-5]. This review focuses on the details of extraction methods, identification and composition of essential oils from the Malaysian Citrus species and also on their biological properties. 


\section{Essential Oils from the Malaysian Citrus Species}

\subsection{Citrus aurantifolia (Cristm.) Swingle}

Citrus aurantifolia (Figure 1), commonly known as 'limau nipis' or 'common lime' is the most popular Citrus species in Malaysia. Usually, C. aurantifolia is used in cuisine and traditional medicine. It is a spiny stem plant which is about $3-5 \mathrm{~m}$ tall. This plant has ovate-shaped $5-9 \mathrm{~cm}$ long leaves with 3-5 cm thickness. The flowers of $C$. aurantifolia are white and the fruits are green and turn yellow after ripe with a diameter of 3-6 cm [6]. The essential oil from C. aurantifolia contains a variety of monoterpene and sesquiterpene hydrocarbons, and limonene (1) is the most abundant one. Traditionally, C. aurantifolia is used to aid digestion process, and to reduce sugar, fat, and cholesterol in blood [7]. The oil extracted from the fruits can be used for cold, asthma, arthritis, and bronchitis [8]. The fruit juice is used as a facial wash to refresh the skin and prevent pimples, increase stamina, treat dysfunctional uterine bleeding, and act as an antidote for poison $[9,10]$. The juice also has been found to be an excellent cough reliever when added with sugar and honey. Moreover, it can also reduce body temperature, remove body smell and act as a softener for meat [6]. Additionally, it also has been useful as mosquito, cat, and moth repellants [11,12]. C. aurantifolia has been reported to have biological activities, e.g., antioxidant and anti-inflammatory [7].

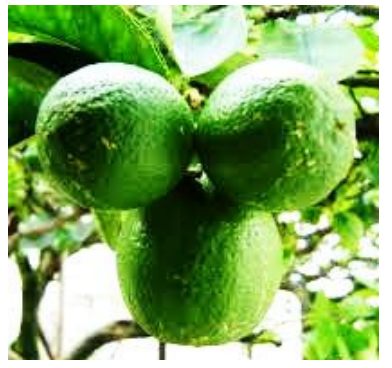

(A)

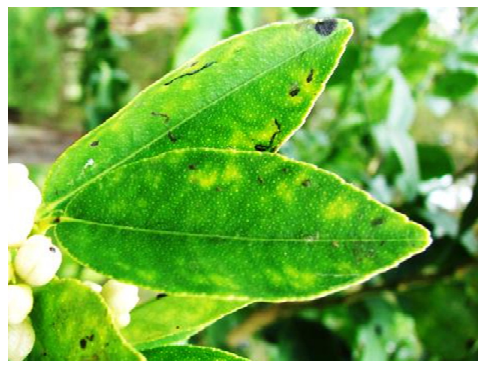

(B)

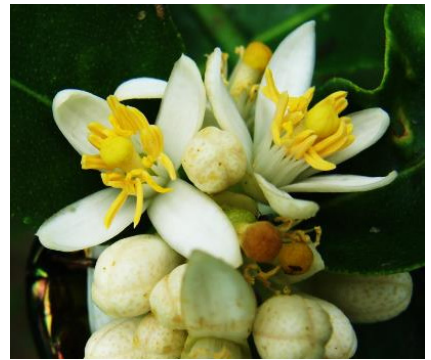

(C)

Figure 1. The (A) fruit; (B) leaf; and (C) flower of C. aurantifolia. (Photos credit: Forest Starr and Kim Starr).

The essential oil of $C$. aurantifolia peels from Masjid Tanah, Melaka, was reported to contain limonene (1) (39.3\%), $\beta$-pinene (2) (28.4\%), geraniol (3) (7.5\%), neral (4) (5.3\%), $\alpha$-terpineol (5) $(2.4 \%)$, geranial (6) (2.1\%), and terpinen-4-ol (7) (2.0\%) (Table 1) [3]. Geranial (6) (19.4\%), limonene (1) (16.4\%), neral (4) (11.4), nerol (8) (9.5\%), geraniol (3) (7.5\%), geranyl acetate (9) (6.6\%), and $\beta$-caryophyllene (10) $(5.7 \%)$ (Figure 2) were the major compounds in the essential oil of the leaves of C. aurantifolia [3].<smiles>C=C(C)C1CC=C(C)CC1</smiles><smiles>C=C1CCC2CC1C2(C)C</smiles>

(2)<smiles>CC(C)=CCCC(C)=CCO</smiles>

(3)<smiles>CC(C)=CCC/C(C)=C\C=O</smiles>

(4)<smiles>CC1=CCC(C(C)(C)O)CC1</smiles>

(5)<smiles>CC(C)=CCCC(C)=CC=O</smiles>

(6)<smiles>CC1=CCC(O)(C(C)C)CC1</smiles>

(7)<smiles>CC(C)=CCC/C(C)=C\CO</smiles>

(8)<smiles>CC(=O)OCC=C(C)CCC=C(C)C</smiles>

(9)<smiles>C=C1CC/C=C(/C)CC[C@H]2C(C)C[C@H]12</smiles>

(10)<smiles>C=CC(=C)CCC=C(C)C</smiles>

(11)<smiles>C=C(C)[C@H]1CC=C(C)[C@H](O)C1</smiles>

(12)

Figure 2. Cont. 
<smiles>CC(=CCO)CCC[C@H](C)CCC[C@H](C)CCCC(C)C</smiles>

(13)<smiles>C=C/C(C)=C\CC=C(C)C</smiles>

(17)<smiles>CC1=CC2CCC=C(CC2)CC1C(C)(C)O</smiles>

(23)<smiles>C=CC(C)(O)CCC=C(C)C</smiles>

(18)<smiles>C=C1CCC2(C(C)C)CC1C2</smiles>

(19)<smiles>CC(C)=CCC[C@H](C)[C@H]1C=CC(C)=CC1</smiles>

(24)<smiles>CC1=C[C@H]2C(C)=CC[C@H](C(C)C)[C@H]2CC1</smiles>

(14)<smiles>C=C/C(C)=C/CC=C(C)C</smiles>

(15)<smiles>CC1=CCC2C(C1)C21CC1</smiles>

(16)<smiles>CC(C)=CCCC(C)CC=O</smiles>

(20)<smiles>CC(C)=CCCC(C)CCO</smiles>

(21)<smiles>CC(=O)OCCC(C)CCC=C(C)C</smiles>

(22)<smiles>CC1=CCC[C@]2(C)CCC(C(C)(C)O)CC12</smiles>

(25)<smiles>C=C1CCC[C@]2(C)CC[C@@H](C(C)(C)O)C[C@H]12</smiles>

(26)

Figure 2. Chemical structures of the components identified from the Citrus essential oils.

\subsection{Citrus grandis L. Osbek}

Citrus grandis L. (synonyms: C. decumana L. and C. maxima Merr.) (Figure 3) is one of the most popular fruits in Malaysia, especially in Tambun, Perak. It is native to Malaysia, and also other countries including Bangladesh, India, Indonesia, Philippines, Thailand, and Vietnam, and grows widely in Malaysia on the tailings of tin mines. In Malaysia, C. grandis has a variety of local names, such as 'limau bali', 'limau abong', 'limau besar', 'limau betawi', 'limau bol' and 'limau jambua'. This plant is also known as 'pumelo' or 'pummelo', with a height of 5-15 $\mathrm{m}$, and the thickness of this plant is 10-30 cm. The leaves of pumelo are dotted, glandular, alternate, ovate and elliptic, 5-20 cm long and 2-12 cm thick. The fruits of pumelo are pear-shaped with a width of 10-30 cm and pale-yellow or greenish yellow color $[13,14]$. C. grandis is well known for its therapeutic values; it can cure fever, gout, arthritis, kidney disorders and ulcers [15]. The fruit pulp and peels are used as an appetizer, stomach-tonic, and also for the treatment of inflammation and cough. The fruit juice has potential in influencing weight loss and promoting cholesterol reduction. In addition, C. grandis fruits are also used in the food, cosmetic, perfume and pharmaceutical industries as a flavoring or fragrance-enhancing agent [16]. The essential oil from the fruits and the leaves of C. grandis is used as one of the components of various toiletry products. Highly aromatic character of its flowers is routinely exploited by perfume manufactures [14].

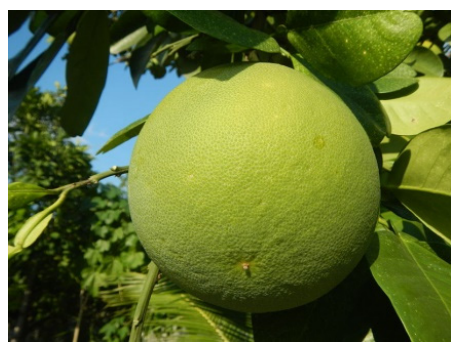

(A)

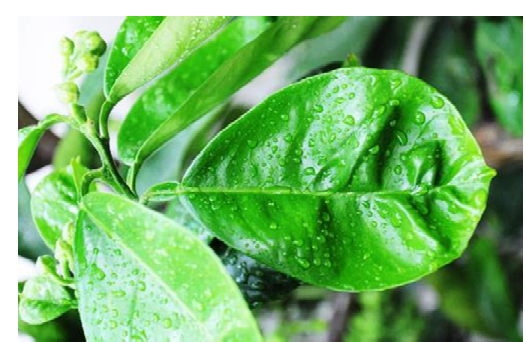

(B)

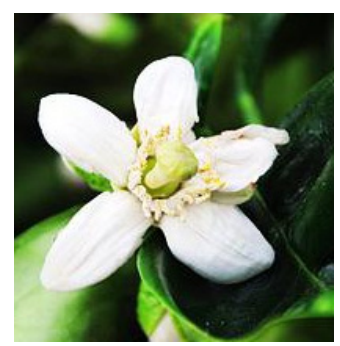

(C)

Figure 3. The (A) fruit; (B) leaf; and (C) flower of C. grandis. (Photos kredit: Judgefloro, Davidals, Amada44). 
Table 1. Composition of Essential Oil of Citrus Species in Malaysia.

\begin{tabular}{|c|c|c|c|c|c|c|c|c|c|c|c|c|}
\hline \multirow[b]{2}{*}{ Plant } & \multirow[b]{2}{*}{ Location } & \multirow[b]{2}{*}{ Method } & \multirow[b]{2}{*}{ Part } & \multicolumn{8}{|c|}{ Components } & \multirow[b]{2}{*}{ Ref. } \\
\hline & & & & $\begin{array}{l}\text { Monoterpene } \\
\text { Hydrocarbon }\end{array}$ & $\%$ & $\begin{array}{c}\text { Oxygenated } \\
\text { Monoterpene }\end{array}$ & $\%$ & $\begin{array}{l}\text { Sesquiterpene } \\
\text { Hydrocarbon }\end{array}$ & $\%$ & $\begin{array}{c}\text { Oxygenated } \\
\text { Sesquiterpene }\end{array}$ & $\%$ & \\
\hline \multirow{12}{*}{$\begin{array}{l}\text { Citrus aurantifolia } \\
\text { (Cristm.) Swingle }\end{array}$} & \multirow{12}{*}{$\begin{array}{l}\text { Masjid } \\
\text { Tanah, } \\
\text { Melaka }\end{array}$} & \multirow{12}{*}{ Water Distillation } & \multirow{6}{*}{ Peel } & $\alpha$-Pinene & 1.5 & Terpinen-4-ol & 2.0 & $\beta$-Caryophyllene & 0.8 & (Z)-Nerolidol & 0.6 & \multirow{12}{*}{ [3] } \\
\hline & & & & $\beta$-Pinene & 28.4 & $\alpha$-Terpineol & 2.4 & $\alpha$-Bergamotene & 0.4 & $\alpha$-Eudesmol & 0.1 & \\
\hline & & & & $\beta$-Myrcene & 1.0 & Neral & 5.3 & $\alpha$-Humulene & 0.1 & $\beta$-Eudesmol & 0.1 & \\
\hline & & & & $\delta$-3-Carene & 0.5 & Geraniol & 7.5 & (Z)- $\beta$-Farnesene & 0.4 & Elemol & 0.1 & \\
\hline & & & & Limonene & 39.3 & Geranial & 2.1 & $(E)-\beta$-Farnesene & 1.5 & & & \\
\hline & & & & $\gamma$-Terpinene & 0.8 & Geranyl acetate & 0.6 & & & & & \\
\hline & & & \multirow{6}{*}{ Leaves } & Sabinene & 0.1 & Linalool & 1.1 & (Z)- $\beta$-Farnesene & 0.1 & Phytol & 1.0 & \\
\hline & & & & $\beta$-Pinene & 0.9 & Nerol & 9.5 & $\alpha$-Humulene & 0.8 & (Z)-Nerolidol & 2.1 & \\
\hline & & & & $\beta$-Myrcene & 0.8 & Neral & 11.4 & $\alpha$-Guaiene & 0.9 & $\alpha$-Eudesmol & 0.3 & \\
\hline & & & & Limonene & 16.4 & Geraniol & 7.5 & $\beta$-Caryophyllene & 5.7 & $\beta$-Eudesmol & 0.3 & \\
\hline & & & & cis- $\beta$-Ocimene & 0.4 & Geranial & 19.4 & $(E)$ - $\beta$-Farnesene & 1.8 & Elemol & 0.5 & \\
\hline & & & & trans- $\beta$-Ocimene & 1.9 & Geranyl acetate & 6.6 & $\beta$-Bisabolene & 0.1 & & & \\
\hline \multirow{24}{*}{$\begin{array}{l}\text { Citrus grandis L. } \\
\text { Osbek }\end{array}$} & \multirow{12}{*}{$\begin{array}{c}\text { Tambun, } \\
\text { Perak }\end{array}$} & \multirow{6}{*}{ Steam Distillation } & \multirow{6}{*}{ Peel } & $\alpha$-Pinene & 0.3 & Linalool & 0.8 & $\delta$-Guaiene & $\operatorname{tr}$ & Caryophyllene oxide & $\operatorname{tr}$ & \multirow{12}{*}{ [4] } \\
\hline & & & & Sabinene & 0.1 & Terpinen-4-ol & 0.1 & $\alpha$-Cubebene & 0.1 & Muurolol & 0.1 & \\
\hline & & & & $\beta$-Pinene & 0.1 & cis-Carveol & 1.5 & trans-Caryophyllene & $\operatorname{tr}$ & Farnesol & 0.3 & \\
\hline & & & & $\beta$-Myrcene & 2.2 & trans-Carveol & 0.6 & Aromadendrene & $\operatorname{tr}$ & Nootkatone & 0.3 & \\
\hline & & & & Limonene & 81.6 & $1-\alpha$-Terpineol & 1.2 & $\delta$-Cadinene & $\operatorname{tr}$ & & & \\
\hline & & & & $\beta$-Cymene & 0.1 & Carvone & 0.9 & & & & & \\
\hline & & \multirow{6}{*}{$\begin{array}{l}\text { Likens-Nikerson } \\
\text { Extraction }\end{array}$} & \multirow{6}{*}{ Peel } & $\alpha$-Pinene & 0.1 & Linalool & 0.5 & 8-Guaiene & $\operatorname{tr}$ & Caryophyllene oxide & $\operatorname{tr}$ & \\
\hline & & & & Sabinene & 0.1 & Terpinen-4-ol & 0.3 & $\alpha$-Cubebene & $\operatorname{tr}$ & Muurolol & $\operatorname{tr}$ & \\
\hline & & & & $\beta$-Pinene & 0.1 & cis-Carveol & 1.4 & trans-Caryophyllene & 0.1 & Farnesol & 0.3 & \\
\hline & & & & $\beta$-Myrcene & 1.6 & trans-Carveol & 0.4 & Aromadendrene & $\operatorname{tr}$ & Nootkatone & 0.3 & \\
\hline & & & & Limonene & 86.8 & 1- $\alpha$-Terpineol & 1.1 & $\delta$-Cadinene & 0.1 & & & \\
\hline & & & & $\beta$-Cymene & $\operatorname{tr}$ & Carvone & 0.7 & & & & & \\
\hline & \multirow{12}{*}{$\begin{array}{l}\text { Kepong, } \\
\text { Selangor }\end{array}$} & \multirow{12}{*}{ Water Distillation } & \multirow{6}{*}{ Peel } & $\beta$-Pinene & 0.6 & Linalool & 0.2 & (Z)- $\beta$-Farnesene & 0.3 & $\alpha$-Eudesmol & $\operatorname{tr}$ & \multirow{12}{*}{ [3] } \\
\hline & & & & $\alpha$-Pinene & 0.3 & Terpinen-4-ol & 0.1 & $\alpha$-Guaiene & 0.1 & Phytol & 0.1 & \\
\hline & & & & $\beta$-Myrcene & 1.6 & $\alpha$-Terpineol & 0.2 & $(E)$ - $\beta$-Farnesene & 0.1 & & & \\
\hline & & & & $\alpha$-Phellandrene & 0.1 & Neral & 0.1 & Aromadendrene & $\operatorname{tr}$ & & & \\
\hline & & & & Limonene & 95.1 & Geraniol & 0.1 & $\beta$-Caryophyllene & 0.1 & & & \\
\hline & & & & $\gamma$-Terpinene & 0.1 & Geranial & 0.1 & & & & & \\
\hline & & & & Sabinene & 0.1 & Terpinolene & 1.6 & $\beta$-Caryophyllene & 15.4 & Hedycaryol & 2.4 & \\
\hline & & & & $\beta$-Pinene & 4.9 & Nerol & 1.5 & $\alpha$-Humulene & 1.8 & (Z)-Nerolidol & 0.8 & \\
\hline & & & & $\delta$-3-Carene & 3.9 & Neral & 4.5 & (Z)- $\beta$-Farnesene & 2.2 & $\alpha$-Eudesmol & 1.8 & \\
\hline & & & Leaves & Limonene & 1.4 & Geraniol & 1.4 & $\alpha$-Cadinene & 7.1 & $\beta$-Eudesmol & 1.6 & \\
\hline & & & & cis- $\beta$-Ocimene & 0.8 & Geranial & 4.5 & $\gamma$-Cadinene & 0.9 & Phytol & 23.1 & \\
\hline & & & & trans- $\beta$-Ocimene & 9.9 & Citronellyl acetate & 1.8 & & & & & \\
\hline
\end{tabular}


Table 1. Cont

\begin{tabular}{|c|c|c|c|c|c|c|c|c|c|c|c|c|}
\hline \multirow[b]{2}{*}{ Plant } & \multirow[b]{2}{*}{ Location } & \multirow[b]{2}{*}{ Method } & \multirow[b]{2}{*}{ Part } & \multicolumn{8}{|c|}{ Components } & \multirow[b]{2}{*}{ Ref. } \\
\hline & & & & $\begin{array}{l}\text { Monoterpene } \\
\text { Hydrocarbon }\end{array}$ & $\%$ & $\begin{array}{c}\text { Oxygenated } \\
\text { Monoterpene }\end{array}$ & $\%$ & $\begin{array}{l}\text { Sesquiterpene } \\
\text { Hydrocarbon }\end{array}$ & $\%$ & $\begin{array}{c}\text { Oxygenated } \\
\text { Sesquiterpene }\end{array}$ & $\%$ & \\
\hline \multirow{3}{*}{$\begin{array}{c}\text { Citrus grandis L. } \\
\text { Osbek } \\
\text { (white pomelo) }\end{array}$} & \multirow{3}{*}{$\begin{array}{l}\text { Tambun, } \\
\text { Perak }\end{array}$} & HS-SPME & Blossom & 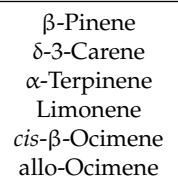 & $\begin{array}{c}0.3 \\
0.6 \\
2.8 \\
48.2 \\
12.0 \\
1.6\end{array}$ & $\begin{array}{c}\text { Linalool } \\
\alpha \text {-Terpineol } \\
\text { Citronellol } \\
\text { Nerol } \\
\text { Geraniol } \\
\text { Carveol }\end{array}$ & $\begin{array}{c}9.2 \\
\text { tr } \\
0.3 \\
1.5 \\
1.3 \\
0.1\end{array}$ & $\begin{array}{c}\beta \text {-Caryophyllene } \\
\alpha \text {-Humulene } \\
\beta \text {-Farnesene } \\
\text { Germacrene D } \\
\beta \text {-Bisabolene } \\
\alpha \text {-Farnesene }\end{array}$ & $\begin{array}{l}0.1 \\
0.1 \\
0.1 \\
0.1 \\
0.1 \\
0.1\end{array}$ & $\begin{array}{c}\text { trans-Nerolidol } \\
\text { cis-Farnesol } \\
\text { Spathulenol }\end{array}$ & $\begin{array}{c}0.1 \\
0.4 \\
\operatorname{tr}\end{array}$ & \\
\hline & & HS-SPME & Peel & $\begin{array}{c}\beta \text {-Pinene } \\
\delta \text {-3-Carene } \\
\alpha \text {-Terpinene } \\
\text { Limonene } \\
\text { cis- } \beta \text {-Ocimene } \\
\beta \text {-Myrcene }\end{array}$ & $\begin{array}{c}0.1 \\
0.1 \\
\text { tr } \\
96.9 \\
0.1 \\
0.2 \\
\end{array}$ & $\begin{array}{c}\text { Linalool } \\
\alpha \text {-Terpineol } \\
\text { Citronellol } \\
\text { Nerol } \\
\text { Geraniol } \\
\text { Carveol }\end{array}$ & $\begin{array}{c}0.2 \\
\text { tr } \\
\text { tr } \\
0.1 \\
0.1 \\
\text { tr }\end{array}$ & $\begin{array}{c}\beta \text {-Caryophyllene } \\
\alpha \text {-Humulene } \\
\beta \text {-Farnesene } \\
\text { Germacrene D } \\
\beta \text {-Bisabolene } \\
\alpha \text {-Farnesene }\end{array}$ & $\begin{array}{c}0.1 \\
\text { tr } \\
0.1 \\
\text { tr } \\
\text { tr } \\
0.1\end{array}$ & $\begin{array}{l}\text { trans-Nerolidol } \\
\text { cis-Farnesol } \\
\text { Elemol }\end{array}$ & $\begin{array}{l}\mathrm{tr} \\
\mathrm{tr} \\
0.1\end{array}$ & [5] \\
\hline & & Solvent extraction & Peel & $\begin{array}{c}\beta \text {-Pinene } \\
\delta \text {-3-Carene } \\
\alpha \text {-Terpinene } \\
\text { Limonene } \\
\text { cis- } \beta \text {-Ocimene } \\
\beta \text {-Myrcene }\end{array}$ & $\begin{array}{c}0.1 \\
0.1 \\
0.1 \\
95.4 \\
0.1 \\
0.1 \\
\end{array}$ & $\begin{array}{c}\text { Linalool } \\
\alpha \text {-Terpineol } \\
\text { Citronellol } \\
\text { Nerol } \\
\text { Geraniol } \\
\text { Carveol }\end{array}$ & $\begin{array}{c}0.2 \\
0.1 \\
\operatorname{tr} \\
0.2 \\
0.3 \\
0.1 \\
\end{array}$ & $\begin{array}{c}\beta \text {-Caryophyllene } \\
\alpha \text {-Humulene } \\
\beta \text {-Farnesene } \\
\text { Germacrene D } \\
\beta \text {-Bisabolene } \\
\alpha \text {-Farnesene }\end{array}$ & $\begin{array}{l}0.1 \\
0.1 \\
0.1 \\
0.1 \\
0.1 \\
0.1\end{array}$ & $\begin{array}{l}\text { trans-Nerolidol } \\
\text { cis-Farnesol } \\
\text { Elemol }\end{array}$ & $\begin{array}{l}0.1 \\
0.1 \\
0.1\end{array}$ & \\
\hline \multirow{3}{*}{$\begin{array}{l}\text { Citrus grandis L. } \\
\text { Osbek } \\
\text { (pink pomelo) }\end{array}$} & \multirow{3}{*}{$\begin{array}{l}\text { Tambun, } \\
\text { Perak }\end{array}$} & $\begin{array}{l}\text { Headspace solid } \\
\text { phase } \\
\text { microextraction } \\
\text { (HS-SPME) }\end{array}$ & Blossom & $\begin{array}{c}\beta \text {-pinene } \\
\delta \text {-3-carene } \\
\alpha \text {-Terpinene } \\
\text { Limonene } \\
\text { cis- } \beta \text {-Ocimene } \\
\text { allo-Ocimene }\end{array}$ & $\begin{array}{c}0.1 \\
0.2 \\
2.5 \\
15.5 \\
4.0 \\
1.1\end{array}$ & $\begin{array}{c}\text { Linalool } \\
\alpha \text {-Terpineol } \\
\text { Citronellol } \\
\text { Nerol } \\
\text { Geraniol } \\
\text { Carveol }\end{array}$ & $\begin{array}{c}56.5 \\
\text { tr } \\
0.2 \\
0.4 \\
0.4 \\
0.1 \\
\end{array}$ & $\begin{array}{c}\beta \text {-Caryophyllene } \\
\alpha \text {-Humulene } \\
\beta \text {-Farnesene } \\
\text { Germacrene D } \\
\beta \text {-Bisabolene } \\
\alpha \text {-Farnesene }\end{array}$ & $\begin{array}{l}0.1 \\
0.1 \\
0.1 \\
0.1 \\
0.1 \\
0.2\end{array}$ & $\begin{array}{c}\text { trans-Nerolidol } \\
\text { cis-Farnesol } \\
\text { Spathulenol }\end{array}$ & $\begin{array}{l}0.1 \\
1.8 \\
\text { tr }\end{array}$ & \multirow{3}{*}{ [5] } \\
\hline & & HS-SPME & Peel & $\begin{array}{c}\beta \text {-Pinene } \\
\delta \text {-3-Carene } \\
\alpha \text {-Terpinene } \\
\text { Limonene } \\
\text { cis- } \beta \text {-Ocimene } \\
\beta \text {-Myrcene }\end{array}$ & $\begin{array}{c}0.1 \\
0.1 \\
\operatorname{tr} \\
96.1 \\
0.1 \\
0.4 \\
\end{array}$ & $\begin{array}{c}\text { Linalool } \\
\alpha \text {-Terpineol } \\
\text { Citronellol } \\
\text { Nerol } \\
\text { Geraniol } \\
\text { Carveol }\end{array}$ & $\begin{array}{c}0.1 \\
0.1 \\
0.1 \\
0.1 \\
0.1 \\
\text { tr }\end{array}$ & $\begin{array}{c}\beta \text {-Caryophyllene } \\
\alpha \text {-Humulene } \\
\beta \text {-Farnesene } \\
\text { Germacrene D } \\
\beta \text {-Bisabolene } \\
\alpha \text {-Farnesene }\end{array}$ & $\begin{array}{c}0.1 \\
\text { tr } \\
0.1 \\
\text { tr } \\
\text { tr } \\
0.1\end{array}$ & $\begin{array}{l}\text { trans-Nerolidol } \\
\text { cis-Farnesol } \\
\text { Elemol }\end{array}$ & $\begin{array}{c}0.1 \\
\operatorname{tr} \\
0.1\end{array}$ & \\
\hline & & Solvent extraction & Peel & $\begin{array}{c}\beta \text {-Pinene } \\
\delta \text {-3-Carene } \\
\alpha \text {-Terpinene } \\
\text { Limonene } \\
\text { cis- } \beta \text {-Ocimene } \\
\beta \text {-Myrcene }\end{array}$ & $\begin{array}{c}0.1 \\
\operatorname{tr} \\
0.1 \\
93.1 \\
0.1 \\
0.1\end{array}$ & $\begin{array}{c}\text { Linalool } \\
\alpha \text {-Terpineol } \\
\text { Citronellol } \\
\text { Nerol } \\
\text { Geraniol } \\
\text { Carveol }\end{array}$ & $\begin{array}{l}0.3 \\
0.2 \\
0.1 \\
0.2 \\
0.3 \\
0.1\end{array}$ & $\begin{array}{c}\beta \text {-Caryophyllene } \\
\alpha \text {-Humulene } \\
\beta \text {-Farnesene } \\
\text { Germacrene D } \\
\beta \text {-Bisabolene } \\
\alpha \text {-Farnesene }\end{array}$ & $\begin{array}{c}0.2 \\
0.1 \\
0.1 \\
0.2 \\
\text { tr } \\
0.1\end{array}$ & $\begin{array}{l}\text { trans-Nerolidol } \\
\text { cis-Farnesol } \\
\text { Elemol }\end{array}$ & $\begin{array}{l}0.1 \\
0.1 \\
0.1\end{array}$ & \\
\hline
\end{tabular}


Table 1. Cont.

\begin{tabular}{|c|c|c|c|c|c|c|c|c|c|c|c|c|}
\hline \multirow[b]{2}{*}{ Plant } & \multirow[b]{2}{*}{ Location } & \multirow[b]{2}{*}{ Method } & \multirow[b]{2}{*}{ Part } & \multicolumn{8}{|c|}{ Components } & \multirow[b]{2}{*}{ Ref. } \\
\hline & & & & $\begin{array}{l}\text { Monoterpene } \\
\text { Hydrocarbon }\end{array}$ & $\%$ & $\begin{array}{c}\text { Oxygenated } \\
\text { Monoterpene }\end{array}$ & $\%$ & $\begin{array}{l}\text { Sesquiterpene } \\
\text { Hydrocarbon }\end{array}$ & $\%$ & $\begin{array}{l}\text { Oxygenated } \\
\text { Sesquiterpene }\end{array}$ & $\%$ & \\
\hline \multirow{7}{*}{ Citrus hystrix D.C. } & \multirow{3}{*}{$\begin{array}{l}\text { Dengkil, } \\
\text { Selangor }\end{array}$} & $\begin{array}{l}\text { Hydro-difusion } \\
\text { steam distillation } \\
\text { system }\end{array}$ & Peel & $\begin{array}{c}\alpha \text {-Thujene } \\
\alpha \text {-Pinene } \\
\text { Sabinene } \\
\beta \text {-Pinene } \\
\beta \text {-Myrcene } \\
\text { Limonene }\end{array}$ & \begin{tabular}{c|}
0.2 \\
1.8 \\
36.4 \\
8.6 \\
1.7 \\
32.5 \\
\end{tabular} & $\begin{array}{c}\text { Linalool } \\
\text { Citronellal } \\
\text { Terpinen-4-ol } \\
\alpha \text {-Terpineol } \\
\text { Citronellol }\end{array}$ & $\begin{array}{c}0.8 \\
10.8 \\
1.1 \\
0.6 \\
1.8\end{array}$ & $\begin{array}{c}\alpha \text {-Copaene } \\
\text { Caryophyllene } \\
\alpha \text {-Humulene } \\
\text { Germacrene D } \\
\beta \text {-Selinene } \\
\delta \text {-Cadinene }\end{array}$ & $\begin{array}{l}0.5 \\
0.3 \\
0.1 \\
0.4 \\
0.1 \\
0.4\end{array}$ & & & [17] \\
\hline & & $\begin{array}{l}\text { Steam distillation } \\
\text { with induction } \\
\text { heating system }\end{array}$ & Peel & $\begin{array}{c}\alpha \text {-Thujene } \\
\alpha \text {-Pinene } \\
\text { Sabinene } \\
\beta \text {-Pinene } \\
\beta \text {-Myrcene } \\
\text { Limonene }\end{array}$ & $\begin{array}{c}0.1 \\
3.2 \\
48.5 \\
10.1 \\
1.5 \\
27.7\end{array}$ & $\begin{array}{c}\text { Linalool } \\
\text { Citronellal } \\
\text { Terpinen-4-ol } \\
\alpha \text {-Terpineol } \\
\text { Citronellyl acetate }\end{array}$ & $\begin{array}{l}0.1 \\
3.3 \\
0.5 \\
0.2 \\
0.1\end{array}$ & $\begin{array}{c}\alpha \text {-Copaene } \\
\text { Germacrene D } \\
\delta \text {-Cadinene }\end{array}$ & $\begin{array}{l}0.1 \\
0.2 \\
0.1\end{array}$ & & & [18] \\
\hline & & $\begin{array}{l}\text { Automated steam } \\
\text { distillation process }\end{array}$ & Peel & $\begin{array}{c}\alpha \text {-thujene } \\
\alpha \text {-pinene } \\
\text { Sabinene } \\
\beta \text {-pinene } \\
\beta \text {-Myrcene } \\
\text { Limonene }\end{array}$ & \begin{tabular}{c|}
0.2 \\
3.3 \\
46.6 \\
13.5 \\
1.8 \\
17.2 \\
\end{tabular} & $\begin{array}{c}\text { Linalool } \\
\text { Citronellal } \\
\text { Terpinen-4-ol } \\
\alpha \text {-Terpineol }\end{array}$ & $\begin{array}{l}1.2 \\
7.8 \\
2.4 \\
0.9\end{array}$ & & & & & [19] \\
\hline & Selangor & Hydro-distillation & Leaves & $\begin{array}{c}\text { Sabinene } \\
\beta \text {-Myrcene } \\
\text { (E)-2,5-Dimethyl-1,6-octadine } \\
\text { cis-2,6-Dimethyl-2,6-octadine }\end{array}$ & $\begin{array}{l}0.2 \\
0.1 \\
0.1 \\
0.3\end{array}$ & $\begin{array}{c}\text { Linalool } \\
\text { Citronellal } \\
\text { Isopregol } \\
\beta \text {-Citronellol } \\
\text { Citronellol } \\
\text { Geraniol }\end{array}$ & $\begin{array}{c}3.9 \\
66.9 \\
0.7 \\
6.6 \\
1.8 \\
0.4 \\
\end{array}$ & & & Nerolidol & 0.1 & [20] \\
\hline & \multirow{3}{*}{$\begin{array}{l}\text { Jerangau, } \\
\text { Terengganu }\end{array}$} & Steam Distillation & $\begin{array}{l}\text { Fresh } \\
\text { Leaves }\end{array}$ & $\begin{array}{c}\alpha \text {-Pinene } \\
\text { Sabinene } \\
\beta \text {-Pinene } \\
\beta \text {-Myrcene } \\
\text { Limonene } \\
p \text {-Cymene }\end{array}$ & $\begin{array}{l}0.1 \\
1.6 \\
0.1 \\
0.7 \\
5.9 \\
0.1\end{array}$ & $\begin{array}{c}\text { Linalool } \\
\text { Citronellal } \\
\beta \text {-Citronellol } \\
\text { iso-Pulegol } \\
\text { Citronellyl acetate }\end{array}$ & $\begin{array}{c}1.0 \\
61.7 \\
13.4 \\
0.9 \\
2.0\end{array}$ & $\begin{array}{c}\text { trans-Caryophyllene } \\
\beta \text {-Elemene } \\
\alpha \text {-Muurolene } \\
\beta \text {-Bisabolene } \\
\delta \text {-Cadinene }\end{array}$ & $\begin{array}{l}\operatorname{tr} \\
\operatorname{tr} \\
\operatorname{tr} \\
\operatorname{tr} \\
\operatorname{tr}\end{array}$ & $\begin{array}{c}\text { Elemol } \\
\text { Nerolidol } \\
\text { Guaiol } \\
\text { Caryophyllene oxide }\end{array}$ & $\begin{array}{c}\operatorname{tr} \\
1.2 \\
0.2 \\
\text { tr }\end{array}$ & [21] \\
\hline & & $\begin{array}{l}\text { Likens-Nikerson } \\
\text { Extraction }\end{array}$ & $\begin{array}{l}\text { Fresh } \\
\text { Leaves }\end{array}$ & $\begin{array}{c}\alpha \text {-Pinene } \\
\text { Sabinene } \\
\beta \text {-Pinene } \\
\beta \text {-Myrcene } \\
\text { Limonene } \\
p \text {-Cymene }\end{array}$ & $\begin{array}{c}0.1 \\
2.0 \\
0.1 \\
0.6 \\
6.8 \\
\text { tr }\end{array}$ & $\begin{array}{c}\text { Linalool } \\
\text { Citronellal } \\
\beta \text {-Citronellol } \\
\text { iso-Pulegol } \\
\text { Citronellyl acetate }\end{array}$ & $\begin{array}{c}1.6 \\
72.5 \\
10.3 \\
1.2 \\
1.2\end{array}$ & $\delta$-Cadinene & $\operatorname{tr}$ & $\begin{array}{l}\text { Elemol } \\
\text { Nerolidol } \\
\text { Guaiol }\end{array}$ & $\begin{array}{l}\operatorname{tr} \\
\operatorname{tr} \\
\operatorname{tr}\end{array}$ & {$[21]$} \\
\hline & & $\begin{array}{l}\text { Likens-Nikerson } \\
\text { Extraction }\end{array}$ & Peel & $\begin{array}{l}\alpha \text {-Pinene } \\
\text { Sabinene } \\
\beta \text {-Pinene } \\
\beta \text {-Myrcene } \\
\text { Limonene } \\
p \text {-cymene }\end{array}$ & $\begin{array}{c}1.7 \\
20.0 \\
23.5 \\
1.0 \\
11.8 \\
0.3\end{array}$ & $\begin{array}{c}\text { Linalool } \\
\text { Citronellal } \\
\beta \text {-Citronellol } \\
\text { iso-pulegol } \\
\text { Citronellyl acetate }\end{array}$ & $\begin{array}{c}1.8 \\
12.6 \\
3.3 \\
0.5 \\
1.7\end{array}$ & $\begin{array}{l}\beta \text {-Bisabolene } \\
\delta \text {-Cadinene }\end{array}$ & $\begin{array}{l}1.2 \\
0.6\end{array}$ & $\begin{array}{c}\text { Elemol } \\
\text { Nerolidol } \\
\text { Guaiol } \\
\text { Caryophyllene oxide }\end{array}$ & $\begin{array}{l}\operatorname{tr} \\
0.2 \\
0.1 \\
0.1\end{array}$ & [21] \\
\hline
\end{tabular}


Table 1. Cont.

\begin{tabular}{|c|c|c|c|c|c|c|c|c|c|c|c|c|}
\hline \multirow[b]{2}{*}{ Plant } & \multirow[b]{2}{*}{ Location } & \multirow[b]{2}{*}{ Method } & \multirow[b]{2}{*}{ Part } & \multicolumn{8}{|c|}{ Components } & \multirow[b]{2}{*}{ Ref. } \\
\hline & & & & $\begin{array}{l}\text { Monoterpene } \\
\text { Hydrocarbon }\end{array}$ & $\%$ & $\begin{array}{c}\text { Oxygenated } \\
\text { Monoterpene }\end{array}$ & $\%$ & $\begin{array}{l}\text { Sesquiterpene } \\
\text { Hydrocarbon }\end{array}$ & $\%$ & $\begin{array}{c}\text { Oxygenated } \\
\text { Sesquiterpene }\end{array}$ & $\%$ & \\
\hline \multirow{12}{*}{ Citrus hystrix D.C. } & \multirow{12}{*}{$\begin{array}{l}\text { Masjid } \\
\text { Tanah, } \\
\text { Melaka }\end{array}$} & \multirow{12}{*}{ Water Distillation } & \multirow{6}{*}{ Peel } & $\alpha$-Pinene & 2.0 & cis-Linalool oxide & 1.9 & $\beta$-caryophyllene & 0.4 & Hedycaryol & 0.3 & \multirow{6}{*}{ [3] } \\
\hline & & & & $\beta$-Pinene & 39.3 & Terpinolene & 1.6 & $\alpha$-Humulene & 0.1 & (Z)-Nerolidol & 0.1 & \\
\hline & & & & Limonene & 14.2 & Linalool & 1.9 & (Z)- $\beta$-Farnesene & 0.2 & $\alpha$-Eudesmol & 0.2 & \\
\hline & & & & $\beta$-Myrcene & 1.3 & Terpinen-4-ol & 8.9 & $\alpha$-Cadinene & 0.1 & $\beta$-Eudesmol & 0.2 & \\
\hline & & & & $\delta$-3-Carene & 1.4 & Citronellal & 11.7 & $(E)-\beta$-Farnesene & 0.1 & Phytol & 0.1 & \\
\hline & & & & $\gamma$-Terpinene & 2.4 & & & $\delta$-Cadinene & 0.5 & $\alpha$-Sinensal & 0.1 & \\
\hline & & & \multirow{6}{*}{ Leaves } & $\beta$-Pinene & 1.9 & $\begin{array}{l}\text { trans-Sabinene } \\
\text { hydrate }\end{array}$ & 1.5 & $\beta$-Cubebene & 0.2 & Hedycaryol & 0.3 & \multirow{6}{*}{ [3] } \\
\hline & & & & $\beta$-Myrcene & 0.9 & Linalool & 1.7 & $\beta$-Caryophyllene & 0.9 & (Z)-Nerolidol & 0.9 & \\
\hline & & & & 8-3-Carene & 0.1 & Citronellal & 72.4 & $\alpha$-Cadinene & 0.4 & $\alpha$-Eudesmol & 0.2 & \\
\hline & & & & Limonene & 0.1 & Citronellol & 6.7 & $\alpha$-Humulene & 0.2 & $\beta$-Eudesmol & 0.2 & \\
\hline & & & & trans- $\beta$-Ocimene & 0.5 & Citronellyl acetate & 4.1 & $(E)-\beta$-Farnesene & 0.2 & Elemol & 0.3 & \\
\hline & & & & $\gamma$-Terpinene & 0.3 & Geranyl acetate & 0.8 & 8-Cadinene & 0.4 & & 0.0 & \\
\hline \multirow{12}{*}{$\begin{array}{l}\text { Citrus microcarpa } \\
\text { (Bunge) Wijnands }\end{array}$} & \multirow{12}{*}{$\begin{array}{c}\text { Masjid } \\
\text { Tanah, } \\
\text { Melaka }\end{array}$} & \multirow{12}{*}{ Water Distillation } & \multirow{6}{*}{ Peel } & $\alpha$-Pinene & 0.5 & $\delta$-Elemene & 0.1 & $\beta$-Caryophyllene & $\operatorname{tr}$ & Elemol & 0.1 & \multirow{6}{*}{ [3] } \\
\hline & & & & $\beta$-Pinene & 0.1 & Linalool & 0.4 & (Z)- $\beta$-Farnesene & 0.7 & $\beta$-Eudesmol & 0.2 & \\
\hline & & & & Myrcene & 1.8 & Terpinen-4-ol & 0.1 & Aromadendrene & 0.1 & & & \\
\hline & & & & $\alpha$-Phellandrene & 0.1 & $\alpha$-Terpineol & 0.3 & $(E)-\beta$-Farnesene & 0.1 & & & \\
\hline & & & & Limonene & 94.0 & Terpinolene & 0.1 & $\alpha$-Guaiene & 0.1 & & & \\
\hline & & & & $\gamma$-Terpinene & 0.1 & Geranyl acetate & 0.2 & & & & & \\
\hline & & & \multirow{6}{*}{ Leaves } & $\alpha$-Pinene & 0.8 & $\delta$-Elemene & 2.7 & $\beta$-Caryophyllene & 2.8 & Hedycaryol & 19.0 & \multirow{6}{*}{ [3] } \\
\hline & & & & $\beta$-Pinene & 13.4 & Linalool & 6.1 & $\alpha$-Humulene & 0.6 & (Z)-Nerolidol & 1.2 & \\
\hline & & & & Myrcene & 0.2 & Terpinen-4-ol & 0.4 & $\alpha$-Sesqui-phellandrene & 18.3 & $\alpha$-Eudesmol & 14.4 & \\
\hline & & & & $\alpha$-Phellandrene & 0.8 & $\alpha$-Terpineol & 0.3 & $\alpha$-Selinene & 1.8 & $\beta$-Eudesmol & 8.6 & \\
\hline & & & & Limonene & 0.7 & $\beta$-Elemene & 1.1 & $\delta$-Cadinene & 0.5 & Elemol & 0.6 & \\
\hline & & & & trans- $\beta$-Ocimene & 2.0 & Geranyl acetate & 0.1 & & & Phytol & 0.4 & \\
\hline
\end{tabular}

tr: trace level. 
Steam distillation method yielded limonene (1) (81.6\%) as the most abundant constituent in the pumelo peel essential oil, while $\beta$-myrcene (11) (2.2\%), and cis-carveol (12) (1.5\%) (Figure 2) were reported as the minor components [4]. However, the Likens-Nikerson method provided slightly higher yield of limonene (1) (86.8\%), followed by $\beta$-myrcene (11) (1.6\%) and cis-carveol (12) (1.4\%) [4]. Comparison of the constituents of pumelo peel oil from Kepong, Selangor, was carried out and limonene (1) (95.1\%) was reported as the major and $\beta$-myrcene (11) $(1.6 \%)$ as the minor components. Sesquiterpene hydrocarbons were identified as the principal components in the leaf essential oil of C. grandis including phytol (13) (23.1\%), $\beta$-caryophyllene (10) (15.4\%) and $\alpha$-cadinene (14) $(7.1 \%)$. Minor monoterpene hydrocarbons were trans- $\beta$-ocimene (15) (9.9\%), $\beta$-pinene (2) (4.9\%), geranial (6) $(4.5 \%)$, and $\delta$-3-carene (16) (3.9\%) (Figure 2) (Table 1) [3]. The essential oil of the fruits peels from the white and the pink pomelo is a major source of limonene (1) (93\%-97\%). Two methods of extraction were employed for the extraction of essential oil from the white and the pink pomelo peels; head-space solid-phase micro-extraction (HS-SPME) and solvent extraction. The essential oil of the blossoms was extracted by the HS-SPME method. The essential oil of the blossoms of white pomelo was found to be rich in limonene (1) (48.2\%), and also contained cis- $\beta$-ocimene (17) (12.0\%), and linalool (18) (9.2\%) (Figure 2). However, the pink pomelo oil was reported to contain an abundant of linalool (18) (56.5\%), and also limonene (1) (15.5\%) and cis- $\beta$-ocimene (17) $(4.0 \%)$ [5].

\subsection{Citrus hystrix}

Citrus hystrix (synonym: kaffir lime) (Figure 4) is known as 'limau purut' or 'wild lime'. C. hystrix leaves and fruits are widely used as spices in preparation of 'tomyam', either white or red, and it is famous dish in Malaysia and Thailand [22]. The height of this plant is about 3-5 $\mathrm{m}$ and the fragrant green leaves are 7.5-10 cm long. It has white flowers with 4-6 petals. The diameter of pear-shaped fruits is about $5.0-7.5 \mathrm{~cm}$ with wrinkle on the surface of fruit. The fruit is dark green, and yellow when ripe [23]. The essential oil of C. hystrix is used in aromatherapy and an essential ingredient of various cosmetic and beauty products [20].

In traditional medicine, C. hystrix is used to treat flu, fever, hypertension, abdominal pains, and diarrhea in infants [24]. The fruits are used as a digestive stimulant, blood purifier, and reduce high blood pressure [25,26]. Additionally, the fruits are used in cooking for flavoring and also in the production of shampoo as an insecticide for washing the head [27]. In addition, the fruit juice is used in softening the skin and the mixture of the fruit juice with bath water can be used to eliminate body odor [28]. Furthermore, the essential oil of C. hystrix has been reported to have various bioactivities such as antioxidant, antibacterial, antileukimic, and antitussive [26].

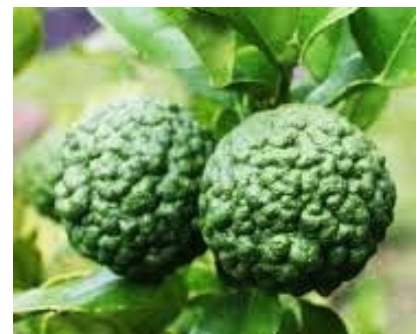

(A)

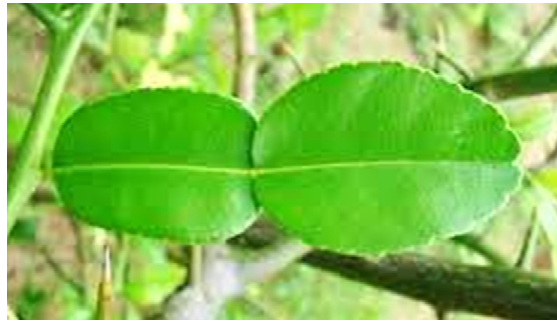

(B)

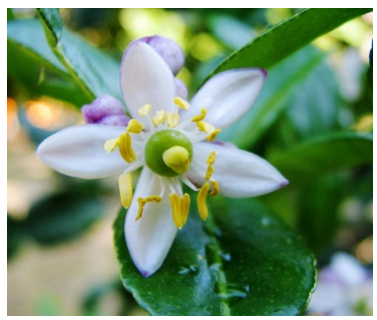

(C)

Figure 4. The (A) fruit; (B) leaf; and (C) flower of C. hystrix. (Photos kredit: Robyn Jay, Forest Starr and Kim Starr, David Rofas).

The essential oil of kaffir lime peel from Dengkil, Selangor, contained sabinene (19) $(36.0 \%-49.0 \%)$, limonene (1) (17.0\%-33.0\%), citronellal (20) (3.0\%-11.0\%) (Figure 2) and $\beta$-pinene (2) (8.0\%-14.0\%) as major components. Three methods were used to extract kaffir lime peel essential oil, e.g., hydro-diffusion steam distillation system, steam distillation with induction heating system, and 
automated steam distillation process with optimized temperature at $90^{\circ} \mathrm{C}$ [17-19]. However, citronellal (20) (66.9\%) and $\beta$-citronellol (21) (6.6\%) (Figure 2) were the major components in kaffir lime peel oil from Selangor, obtained using the hydro-distillation method [29].

The essential oil of $C$. hystrix fresh leaves from Jerangau, Terengganu, extracted by the steam distillation and the Likens-Nikerson extraction methods, was found to be dominated by citronellal (20) $(61.0 \%-73.0 \%)$, $\beta$-citronellol (21) (10.0\%-14.0\%), and limonene (1) (5.0\%-7.0\%) as major components (Table 1). $\beta$-Pinene (2) (23.5\%) and sabinene (19) (20.1\%) appeared as the major components of C. hystrix peel, followed by citronellal (20) (12.6\%), limonene (1) (11.8\%), and $\beta$-citronellol (21) (3.3\%) [21]. Moreover, $\beta$-pinene (2) (39.3\%), limonene (1) (14.2\%), citronellal (20) (11.7\%), and terpinen-4-ol (7) $(8.9 \%)$ were identified as the principal components in kaffir lime peels from Masjid Tanah, Melaka. However, citronellal (20) (72.4\%), $\beta$-citronellol (21) (6.7\%), and citronellyl acetate (22) (4.1\%) (Figure 2) were reported to be the major components in kaffir lime leaves, followed by $\beta$-pinene (2) (1.9\%) and limonene (1) $(0.1 \%)$ as minor components. Water distillation was used as a method to extract kafir lime peels and leaves from Masjid Tanah, Melaka [3].

The antibacterial susceptibility of the essential oils and oil emulsions of Malaysian C. hystrix was evaluated against Escherichia coli, Bacillus subtilis, and Staphylococcus aureus using the disc diffusion method. Pure essential oil with a percentage of $2 \%$ by weight exhibited a strong inhibitory effect against E. coli and B. subtilis with the zones of inhibition of 16.0 and $15.0 \mathrm{~mm}$, respectively. Meanwhile, the formulated emulsions with surfactant mixture of Tween 80 and Span 80 (90:10) with 2\% (by weight) essential oil displayed the most potential antibacterial activity against $E$. coli with the zones of inhibition ranging between 11.0 to $18.0 \mathrm{~mm}$ [2].

The topical application bioassay on uniform weighted second instar larvae in the laboratory was carried out to determine the insecticidal properties of the essential oil from the leaves of the Malaysian C. hystrix against Spodoptera litura (tobacco army worm). The study demonstrated considerable repellant activity of $C$. hystrix essential oil against the $S$. litura larvae after 24 and $48 \mathrm{~h}$ of treatment with $\mathrm{LD}_{50}$ values of 29.25 and $26.75 \mu \mathrm{g} / \mathrm{mL}$, respectively [29].

\subsection{Citrus microcarpa (Bunge) Wijnands}

Citrus microcarpa (synonym: C. madurensis) (Figure 5), common name: 'limau kasturi' in Malaysia, is used in the preparation of beverages. C. microcarpa is $3-5 \mathrm{~m}$ tall with abundant of long spine on the stem, branches and twigs. The dark green leaves of $C$. microcarpa are between $2.5-6.8 \mathrm{~cm}$ long and $2-3 \mathrm{~cm}$ thick. The round or oblong-shaped green leaves of this plant are $2.5-3.8 \mathrm{~cm}$ in diameter. This plant is used to treat fever, cough, and pharyngitis [30]. The juice is traditionally used to prevent respiratory diseases, strengthen the bones and act as growth stimulant for children. The juice is also commonly used in cooking as flavoring ingredients and additives. The leaves of this plant can be used in the treatment of skin diseases, relieve headache and also act as a mouth wash to treat sore throat [31]. Essential oil from C. microcarpa is used commercially in perfumes, food, cosmetics and detergents. It is one of the ingredients in pharmaceutical, aromatherapy and antiseptic products [32].

The essential oil from C. microcarpa peels was reported to be rich in limonene (1) (94.0\%) similar to C. aurantifolia. $\beta$-Myrcene (11) (1.8\%), linalool (18) (0.4\%), and $\alpha$-terpineol (5) (0.3\%) were detected as the minor components (Table 1) in the oil. Sesquiterpene hydrocarbons were the most abundant in the leaves of C. microcarpa. These include hedycaryol (23) (19.0\%), $\alpha$-sesquiphellandrene (24) (18.3\%), $\alpha$-eudesmol (25) (14.4\%) and $\beta$-eudesmol (26) (8.6\%) (Figure 2). The essential oil was extracted by hydrodistillation for $8 \mathrm{~h}$ similar to that of C. grandis and C. aurantifolia oils [3]. 


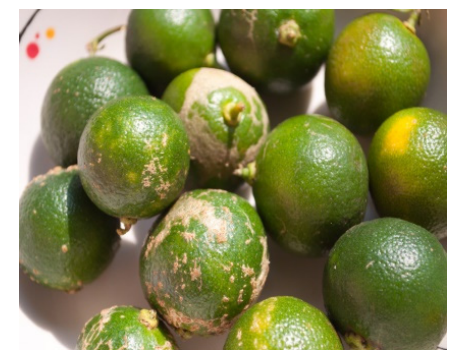

(A)

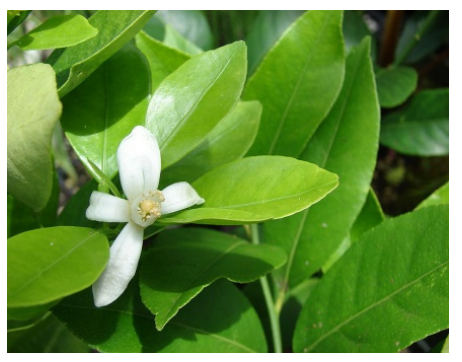

(B)

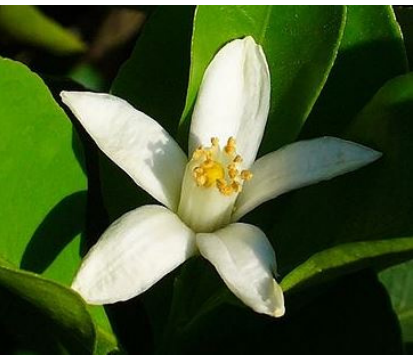

(C)

Figure 5. The (A) fruit; (B) leaf and (C) flower of C. microcarpa. (Photos kredit: Ronald Escanlar, Forest Starr and Kim Starr, H. Zell).

\section{Conclusions}

Extraction and identification of the essential oils from the Malaysian Citrus species showed that limonene (1) (96.9\%) and sabinene (19) (48.5\%) were the major components in C. grandis and C. hystrix, respectively. Sample collections from different locations, and differences in extraction methods resulted in different composition and percentage of yields. Moreover, extraction of essential oils from different parts of Citrus plants also gave different major components. The bioactivity studies on the C. hystrix essential oil revealed strong antimicrobial activity against $E$. coli and good antifeedant properties against S. litura. More bioactivity studies on the essential oils of the Malaysian Citrus plants need to be carried out to acquire better bioactivity profiles of these oils.

Acknowledgments: The authors acknowledge the Ministry of Higher Education (MOHE) for financial support under the Research Universiti Grant (RUG) with vote number Q.J130000.2526.12H36 and the Faculty of Science, Universiti Teknologi Malaysia for providing the necessary support for this study.

Author Contributions: All authors contributed equally, and have given final approval of the version submitted.

Conflicts of Interest: The authors declare no conflict of interest.

\section{References}

1. Manner, H.I.; Buker, R.S.; Smith, V.E.; Ward, D.; Elevitch, C.R. Citrus (citrus) and Fortunella (kumquat). Species Profile Pac. Isl. Agrofor. 2006, 2, 1-35.

2. Ng, D.S.H.; Rose, L.C.; Suhaimi, H.; Mohammad, H.; Rozaini, M.Z.H.; Taib, M. Preliminary evaluation on the antibacterial activities of Citrus hystrix oil emulsions stabilized by tween 80 and span 80. Int. J. Pharm. Pharm. Sci. 2011, 3, 209-211.

3. Jantan, I.; Ahmad, A.S.; Ahmad, A.R.; Ali, N.A.M.; Ayop, N. Chemical composition of some Citrus oils from Malaysia. J. Essent. Oil Res. 1996, 8, 627-632. [CrossRef]

4. Nor, O.M. Sebatian aroma dalam minyak Citrus grandis (Aromatic compounds from Citrus grandis Oil). J. Trop. Agric. Food Sci. 1999, 27, 231-236.

5. Cheong, M.-W.; Loke, X.-Q.; Liu, S.-Q.; Pramudya, K.; Curran, P.; Yu, B. Characterization of volatile compounds and aroma profiles of Malaysian pomelo (Citrus grandis (L.) Osbeck) blossom and peel. J. Essent. Oil Res. 2011, 23, 34-44. [CrossRef]

6. Saidan, I. Dalam Dusun Melayu (in Malay Orchard); Dewan Bahasa dan Pustaka: Kuala Lumpur, Malaysia, 2013; pp. 243-244.

7. Samah, B. Serangan Jantung: Punca, Pencegahan E Kaedah Meredakannya. (Heart Attack: The Cause, Prevention $\mathcal{E}$ Treatment); Alaf 21: Selangor, Malaysia, 2009; pp. 104-105.

8. Kunow, M.A. Maya Medicine: Traditional Healing in Yucatan; University of New Mexico Press: Albuquerque, NM, USA, 2003; p. 117.

9. Aibinu, I.; Adenipekun, T.; Adelowotan, T.; Ogunsanya, T.; Odugbemi, T. Evaluation of the antimicrobial properties of different parts of Citrus aurantifolia (lime fruit) as used locally. Afr. J. Tradit. Complemnet. Altern. Med. 2007, 4, 185-190.

10. Khare, C.P. Indian Medicinal Plants: An Illustrated Dictionary; Springer: Berlin, Germany, 2007; p. 154. 
11. Effiom, O.E.; Avoaja, D.A.; Ohaeri, C.C. Mosquito repellent activity of phytochemical extracts from fruit peels of Citrus fruit species. Glob. J. Sci. Front. Res. 2012, 12, 5-8.

12. Lyle, S. How to Use Citrus Fruit peels in the Home and Garden. In Discovering Fruit and Nuts; David Bateman Ltd.: Auckland, New Zealand, 2006; pp. 130-142.

13. Morton, J.F. Fruits of Warm Climates; Creative Resource Systems, Inc.: Winterville, NC, USA, 1987; pp. $147-151$.

14. Roger, G.D.P. Encyclopedia of Medicinal Plants Education And Health, 1st ed.; Library editorial safeliz SL: Malaga, Spain, 2002; pp. 153-154.

15. Orwa, C.; Mutua, A.; Kindt, R.; Jamnadass, R.; Simons, A. Agroforestree Database: A Tree Reference and Selection Guide Version 4.0. 2009. Available online: http://www.worldagroforestry.org/af/treedb/ (accessed on 15 February 2011).

16. Thavanapong, N.; Wetwitayaklung, P.; Charoenteeraboon, J. Comparison of essential oils compositions of Citrus maxima Merr. peel obtained by cold press and vacuum stream distillation methods and of its peel and flower extract obtained by supercritical carbon dioxide extraction method and their antimicrobial activity. J. Essent. Oil Res. 2010, 22, 71-77.

17. Mohd-Yusoff, Z.; Muhammad, Z.; Kasuan, N.; Rahiman, M.H.F.; Taib, M.N. Effect of temperature on kaffir lime oil by using Hydro-diffusion steam distillation system. Malays. J. Anal. Sci. 2013, 17, 326-339.

18. Muhammad, Z.; Mohd Yusoff, Z.; Nordin, M.N.N.; Kasuan, N.; Taib, M.N.; Rahiman, M.H.F.; Haiyee, Z.A. Steam distillation with induction heating system: Analysis of Kaffir Lime oil compound and production yield at various temperatures. Malays. J. Anal. Sci. 2013, 17, 340-347.

19. Kasuan, N.; Muhammad, Z.; Yusoff, Z.; Rahiman, M.H.F.; Taib, M.N.; Haiyee, Z.A. Extraction of Citrus hystrix DC (Kaffir Lime) essential oil using automated steam distillation process: Analysis of volatile compounds. Malays. J. Anal. Sci. 2013, 17, 359-369.

20. Waikedre, J.; Dugay, A.; Barrachina, I.; Herrenknecht, C.; Cabalion, P.; Fournet, A. Chemical composition and antimicrobial activity of the essential oils from New Caledonian Citrus macroptera and Citrus hystrix. Chem. Biodivers. 2010, 7, 871-877. [CrossRef] [PubMed]

21. Nor, O.M. Volatile aroma compounds in Citrus hystrix oil. J. Trop. Agric. Food Sci. 1999, 27, 225-229.

22. Karim, R.A. Limau Purut Cantikkan Kulit (Skin care by kaffir lime). Utusan Malaysia. 2002. Available online: ww1.utusan.com.my/utusan/info.asp? $y=2002 \& \mathrm{dt}=0317 \&$ pub=Utusan_Malaysia\&sec=Kesihatan\& pg=kn_06.htm (accessed on 17 March 2002).

23. Awang, C.R.C. Kesan Minyak Esensial Citrus spp Ke Atas Sistem Kardiovaskular Tikus Serta Kesan Antiresah dan Antidepresi Pada Mencit. (Anti-Anxious and Anti-Depression Effects of Citrus spp Essential Oils towards Cardiovascular System of Mice). Master's Thesis, Universiti Sains Malaysia, Pulau Pinang, Malaysia, 1 May 2007.

24. Fortin, H.; Vigora, C.; Lohezic-Le, F.; Robina, V.; le Bosse, B.; Boustiea, J.; Arnoros, M. In vitro antiviral activity of thirty-six plants from La Reunion Island. Fitoterapia 2002, 73, 346-350. [CrossRef]

25. Dasuki, S. 202 Khasiat Herba (202 Benefits of Herbs); Grup Buku Karangkraf: Selangor, Malaysia, 2011; pp. 222-223.

26. Norkaew, O.; Pitija, K.; Pripdeevech, P.; Sookwong, P.; Wongpornchai, S. Supercritical fluid extraction and gas chromatographic-mass spectrometric analysis of terpenoids in fresh Kaffir lime leaf oil. Chiang Mai J. Sci. 2013, 40, 240-247.

27. Koh, D.; Ong, C.N. Phytophotodermatitis due to the application of Citrus hystrix as a folk remedy. Br. J. Dermatol. 1999, 140, 737-738. [CrossRef] [PubMed]

28. Dassanayake, M.D. A Revised Handbook to the Flora of Ceylon. Vol. V; Amerind Publishing Co Ltd.: New Delhi, India, 1985; pp. 432-433.

29. Loh, F.S.; Awang, R.M.; Omar, D.; Rahmani, M. Insecticidal properties of Citrus hystrix DC leaves essential oil against Spodoptera litura fabricius. J. Med. Plants Res. 2011, 5, 3739-3744.

30. Ong, H.C. Buah: Khasiat Makanan \& Ubatan; Utusan Publications \& Distributors: Kuala Lumpur, Malaysia, 2004; p. 90.

31. Morton, J.F. Mexican Lime. In Fruits of Warm Climates, 1st ed.; Creative Resource Systems: Winterville, NC, USA, 1987; pp. 168-172.

32. Manaf, Y.N.; Osman, A.; Lai, O.M.; Long, K.; Ghazali, H.M. Characterisation of musk lime (Citrus microcarpa) seed oil. J. Sci. Food Agric. 2008, 88, 676-683. [CrossRef]

(C) 2016 by the authors; licensee MDPI, Basel, Switzerland. This article is an open access article distributed under the terms and conditions of the Creative Commons Attribution (CC-BY) license (http:/ / creativecommons.org/licenses/by/4.0/). 